\title{
ANALYSIS OF FINITE ELEMENT METHODS FOR LINEAR HYPERBOLIC PROBLEMS
}

\author{
RICHARD S. FALK ${ }^{\star}$ \\ Department of Mathematics \\ Rutgers University \\ Piscataway, NJ 08854 \\ falk@math. rutgers.edu
}

\begin{abstract}
We summarize several techniques of analysis for finite element methods for linear hyperbolic problems, illustrating their key properties on the simplest model problem. These include the discontinuous Galerkin method, the continuous Galerkin methods on rectangles and triangles, and a nonconforming linear finite element on a special triangular mesh.
\end{abstract}

\section{Introduction}

Let $\Omega$ be a bounded polygonal domain and consider the simple linear hyperbolic problem:

$$
\boldsymbol{\alpha} \cdot \nabla u=f \quad \text { in } \Omega, \quad u=g \quad \text { on } \Gamma_{\text {in }}(\Omega),
$$

where $\boldsymbol{\alpha}=\left(\alpha_{1}, \alpha_{2}\right)$ is a constant vector and $\Gamma_{i n}(\Omega)$ is the portion of the boundary of $\Omega$ on which $\boldsymbol{\alpha} \cdot \boldsymbol{n}<0$, with $\boldsymbol{n}$ denoting the unit outward normal to $\partial \Omega$.

In this paper, we review several finite element methods proposed for this model problem, and discuss the key ingredients of their analysis. At the most basic level, all of the numerical analysis tries to follow in some way the basic conservation property of the homogeneous equation. That is, multiplying the homogeneous equation by $u$ and integrating over a subdomain $G$, we have

$$
0=(\boldsymbol{\alpha} \cdot \nabla u, u)_{G}=\frac{1}{2} \int_{G} \boldsymbol{\alpha} \cdot \nabla\left(u^{2}\right)=\frac{1}{2} \int_{\partial G} u^{2} \boldsymbol{\alpha} \cdot \boldsymbol{n} .
$$

This may be written in the form

$$
\frac{1}{2} \int_{\Gamma_{\text {out }}(G)} u^{2}|\boldsymbol{\alpha} \cdot \boldsymbol{n}|=\frac{1}{2} \int_{\Gamma_{\text {in }}(G)} u^{2}|\boldsymbol{\alpha} \cdot \boldsymbol{n}|
$$

since $\boldsymbol{\alpha} \cdot \boldsymbol{n} \geq 0$ on $\Gamma_{\text {out }}(G)$ and $\boldsymbol{\alpha} \cdot \boldsymbol{n} \leq 0$ on $\Gamma_{\text {in }}(G)$.

If we choose $\Omega$ so that it is the disjoint union of subdomains $G_{i}$ and sum these identities, cancellation of integrals over the common boundaries leads to the conservation result:

$$
\frac{1}{2} \int_{\Gamma_{\text {out }}(\Omega)} u^{2}|\boldsymbol{\alpha} \cdot \boldsymbol{n}|=\frac{1}{2} \int_{\Gamma_{\text {in }}(\Omega)} u^{2}|\boldsymbol{\alpha} \cdot \boldsymbol{n}| .
$$

It is this type of analysis that we wish to follow at the discrete level to obtain stability and an error analysis of finite element approximation schemes.

\footnotetext{
* This work was supported by NSF grant DMS-9704556.
} 


\section{The Discontinuous Galerkin Method}

We begin with the method which is the subject of this conference, and whose analysis is the most familiar. Let $\tau_{h}$ denote a triangulation of $\Omega$ into triangles $T$ of diameter $\leq h$ and $P_{n}(T)$ the space of polynomials of degree $\leq n$ on $T$. For each $T \in \tau_{h}$, the discontinuous Galerkin method is:

Find $u_{h} \in P_{n}(T)$ such that

$$
\left(\boldsymbol{\alpha} \cdot \nabla u_{h}, v_{h}\right)_{T}-\int_{\Gamma_{i n}(T)} J\left[u_{h}\right] v_{h} \boldsymbol{\alpha} \cdot \boldsymbol{n}=\left(f, v_{h}\right)_{T}
$$

for all $v_{h} \in P_{n}(T)$, where $J[v]=v^{+}-v^{-}$, with $v^{ \pm}(x)=\lim _{\epsilon \rightarrow 0^{ \pm}} v(x+\epsilon \boldsymbol{\alpha})$, $u_{h}^{-}(x)=g(x)$ if $x \in \Gamma_{i n}(\Omega)$, and $(\cdot, \cdot)_{T}$ denotes the $L^{2}$ inner product over $T$.

For this method, one can follow the lead of the continuous problem and take the test function $v_{h}=u_{h}$. Then for the homogeneous problem $f=0$,

$$
\left(\boldsymbol{\alpha} \cdot \nabla u_{h}, u_{h}\right)_{T}-\int_{\Gamma_{i n}(T)} J\left[u_{h}\right] u_{h}^{+} \boldsymbol{\alpha} \cdot \boldsymbol{n}=0 .
$$

Integrating the first term by parts as before and recombining terms, one gets

$$
\begin{aligned}
\frac{1}{2} \int_{\Gamma_{\text {out }}(T)}\left(u_{h}^{-}\right)^{2}|\boldsymbol{\alpha} \cdot \boldsymbol{n}| & =\frac{1}{2} \int_{\Gamma_{\text {in }}(T)}\left(u_{h}^{+}\right)^{2}|\boldsymbol{\alpha} \cdot \boldsymbol{n}|-\int_{\Gamma_{\text {in }}(T)}\left[u_{h}^{+}-u_{h}^{-}\right] u_{h}^{+}|\boldsymbol{\alpha} \cdot \boldsymbol{n}| \\
& =\frac{1}{2} \int_{\Gamma_{\text {in }}(T)}\left(u_{h}^{-}\right)^{2}|\boldsymbol{\alpha} \cdot \boldsymbol{n}|-\frac{1}{2} \int_{\Gamma_{\text {in }}(T)}\left(J\left[u_{h}\right]\right)^{2}|\boldsymbol{\alpha} \cdot \boldsymbol{n}| .
\end{aligned}
$$

Summing over all triangles in the triangulation comprising $\Omega$ gives

$$
\frac{1}{2} \int_{\Gamma_{\text {out }}(\Omega)}\left(u_{h}^{-}\right)^{2}|\boldsymbol{\alpha} \cdot \boldsymbol{n}|+\sum_{T} \frac{1}{2} \int_{\Gamma_{\text {in }}(T)}\left(J\left[u_{h}\right]\right)^{2}|\boldsymbol{\alpha} \cdot \boldsymbol{n}|=\frac{1}{2} \int_{\Gamma_{\text {in }}(\Omega)} g^{2}|\boldsymbol{\alpha} \cdot \boldsymbol{n}| .
$$

This identity is of course the basic one needed to establish stability of the method. The additional test function $\boldsymbol{\alpha} \cdot \nabla u_{h}$, used by Johnson and Pitkäranta [4] provides additional stability and leads to an improvement in the error estimates originally obtained by Lesaint and Raviart [5].

\section{Winther's method}

Next consider a method proposed by R. Winther, using a rectangular mesh. The approximate solution is sought in the space of continuous tensor product piecewise polynomials of degree $\leq n$ in each variable. On each rectangle $R$, the approximate solution $u_{h} \in \boldsymbol{Q}_{n}$ is determined by:

$$
\left(\boldsymbol{\alpha} \cdot \nabla u_{h}, v_{h}\right)_{R}=\left(f, v_{h}\right)_{R} \quad \text { for all } v_{h} \in \boldsymbol{Q}_{n-1},
$$

where $\boldsymbol{Q}_{n}$ denotes the space of tensor products of polynomials of degree $n$ in $x$ and $y$. These equations must be solved in an order determined by the characteristic direction 
and it is assumed that $u_{h}$ is already known on the inflow boundary of the rectangle $R$. In the simplest case $n=1, u_{h}$ is known at 3 of the rectangle vertices and the value at the fourth is determined by taking inner products against constants. In this case, the method is the box scheme or a simple finite volume method.

The analysis of such a method is not so obvious, since the test function $v_{h}=u_{h}$ is not allowed. Winther's idea is to get conservation of a quantity equivalent to $L^{2}$ conservation of $u$ by choosing two test functions. The first is $\left(u_{h}\right)_{x y}$. Considering the homogeneous problem, and dropping the subscript $h$ for the moment, we have for the rectangle $R_{i j}$ with corners $(i h, j h),([i+1] h, j h),(i h,[j+1] h)$, and $([i+$ $1] h,[j+1] h)$,

$$
\begin{aligned}
\left(\alpha_{1} u_{x}+\right. & \left.\alpha_{2} u_{y}, u_{x y}\right)_{R_{i j}}=\frac{1}{2} \int_{i h}^{[i+1] h} \int_{j h}^{[j+1] h}\left(\alpha_{1}\left[\left(u_{x}\right)^{2}\right]_{y}+\alpha_{2}\left[\left(u_{y}\right)^{2}\right]_{x}\right) d y d x \\
= & \frac{1}{2} \int_{i h}^{[i+1] h} \alpha_{1}\left\{\left[u_{x}(x,[j+1] h)\right]^{2}-\left[u_{x}(x, j h)\right]^{2}\right\} d x \\
& \quad+\frac{1}{2} \int_{j h}^{[j+1] h} \alpha_{2}\left\{\left[u_{y}([i+1] h, y)\right]^{2}-\left[u_{y}(i h, y)\right]^{2}\right\} d y \\
= & \frac{1}{2} \int_{\Gamma_{\text {out }}\left(R_{i j}\right)}\left(u_{\tau}\right)^{2}|\boldsymbol{\alpha} \cdot \boldsymbol{s}|-\frac{1}{2} \int_{\Gamma_{i n}\left(R_{i j}\right)}\left(u_{\tau}\right)^{2}|\boldsymbol{\alpha} \cdot \boldsymbol{s}|,
\end{aligned}
$$

where $s$ denotes the unit tangent vector to $\partial R_{i j}$ and $u_{\tau}=\nabla u \cdot s$ denotes the tangential derivative along $\partial R_{i j}$.

Since $u_{h}$ is continuous across rectangle edges, so is $\left(u_{h}\right)_{\tau}$, so summing over all rectangles leads to cancellations and the following result.

$$
\frac{1}{2} \int_{\Gamma_{\text {out }}(\Omega)}\left[\left(u_{h}\right)_{\tau}\right]^{2}|\boldsymbol{\alpha} \cdot \boldsymbol{s}|=\frac{1}{2} \int_{\Gamma_{\text {in }}(\Omega)}\left[\left(u_{h}\right)_{\tau}\right]^{2}|\boldsymbol{\alpha} \cdot \boldsymbol{s}| .
$$

Since this is only a seminorm for functions defined on these boundaries, Winther considered a second test function, which is closer to the spirit of the original analysis. Letting $P_{x}$ and $P_{y}$ denote $L^{2}$ projections into polynomials of degree $n-1$ in $x$ and $y$, respectively, Winther used the test function $v_{h}=P_{x, y} u_{h}$, where $P_{x, y} \equiv P_{x} P_{y}$. Again dropping the subscript $h$, we have

$$
\begin{aligned}
0= & \left(\alpha_{1} u_{x}+\alpha_{2} u_{y}, P_{x, y} u\right)=\left(\alpha_{1} u_{x}, P_{y} u\right)+\left(\alpha_{2} u_{y}, P_{x} u\right) \\
= & \left(\alpha_{1}\left[P_{y} u\right]_{x}, P_{y} u\right)+\left(\alpha_{2}\left[P_{x} u\right]_{y}, P_{x} u\right) \\
= & \frac{1}{2} \int_{i h}^{[i+1] h} \int_{j h}^{[j+1] h}\left(\alpha_{1}\left(\left[P_{y} u\right]^{2}\right)_{x}+\alpha_{2}\left(\left[P_{x} u\right]^{2}\right)_{y}\right) d y d x \\
= & \frac{1}{2} \int_{j h}^{[j+1] h} \alpha_{1}\left\{\left[P_{y} u([i+1] h, y)\right]^{2}-\left[P_{y} u(i h, y)\right]^{2}\right\} d y \\
& \quad+\frac{1}{2} \int_{i h}^{[i+1] h} \alpha_{2}\left\{\left[P_{x} u(x,[j+1] h)\right]^{2}-\left[P_{x} u(x, j h)\right]^{2}\right\} d x \\
= & \frac{1}{2} \int_{\Gamma_{\text {out }}\left(R_{i j}\right)}\left(P_{0} u\right)^{2}|\boldsymbol{\alpha} \cdot \boldsymbol{n}|-\frac{1}{2} \int_{\Gamma_{\text {in }}\left(R_{i j}\right)}\left(P_{0} u\right)^{2}|\boldsymbol{\alpha} \cdot \boldsymbol{n}|,
\end{aligned}
$$


where now $P_{0}$ represents $L^{2}$ projection into polynomials of degree $n-1$ along an edge. Adding $h^{2}$ times the first identity to the second identity gives conservation of a norm that is equivalent on piecewise polynomials to the $L^{2}$ norm.

\section{The Continuous Galerkin method on triangles}

This method was originally proposed by Reed and Hill [6] and was analyzed in [2]. Here we note that are two types of triangles: type I triangles with one inflow side and type II triangles with two inflow sides. We then seek an approximate solution in the space of continuous piecewise polynomials of degree $\leq n$ determined on each triangle $T$ by the variational equations

$$
\left(\boldsymbol{\alpha} \cdot \nabla u_{h}, v_{h}\right)_{T}=\left(f, v_{h}\right)_{T}
$$

where $v_{h} \in P_{n-1}(T)$ on a type I triangle and $v_{h} \in P_{n-2}(T)$ on a type II triangle. By continuity, $u_{h}$ will already be known at $n+1$ degrees of freedom on a type I triangle and at $2 n+1$ degrees of freedom on a type II triangle. Since the total number of degrees of freedom for polynomials of degree $\leq n$ is equal to $(n+1)(n+2) / 2$, and

$$
(n+1)(n+2) / 2=n(n+1) / 2+(n+1)=(n-1) n / 2+(2 n+1),
$$

we have the same number of equations as unknows on both types of triangles. Once again, the analysis of this method is not so obvious, since the simple test function $v_{h}=u_{h}$ is not allowed. However, Winther's analysis gives a clue and Richter noticed that the analogue of $u_{x y}$ for triangles is the choice $v=u_{\tau_{1} \tau_{2}}$, with $\tau_{1}$ and $\tau_{2}$ chosen to be the variables along the two inflow sides of a type II triangle or two outflow sides of a type I triangle. This leads to the following useful identity.

$$
\begin{aligned}
-\int_{T}(\boldsymbol{\alpha} \cdot \nabla u)\left(u_{\tau_{1} \tau_{2}}\right)=\frac{1}{2} & \int_{\partial T} \frac{\left(\boldsymbol{\alpha} \cdot \boldsymbol{n}_{1}\right)\left(\boldsymbol{\alpha} \cdot \boldsymbol{n}_{2}\right)}{\boldsymbol{\alpha} \cdot \boldsymbol{n}} u_{\tau}^{2} \\
& -\frac{1}{2} \int_{\Gamma_{3}} \frac{\left(\tau_{1} \cdot \boldsymbol{n}_{3}\right)\left(\tau_{2} \cdot \boldsymbol{n}_{3}\right)}{\boldsymbol{\alpha} \cdot \boldsymbol{n}_{3}}(\boldsymbol{\alpha} \cdot \nabla u)^{2},
\end{aligned}
$$

where $\Gamma_{3}$ is the inflow side of a type I triangle and the outflow side of a type II triangle, and $\boldsymbol{n}_{i}$ is the unit outward normal to $\Gamma_{i}$. Once again, the continuity of $u_{h}$ ensures the continuity of $\left(u_{h}\right)_{\tau}$ across triangle edges and when this identity is summed over the triangles, cancellations occur on the triangle boundaries which lead to a stability result. For the homogeneous problem, $\boldsymbol{\alpha} \cdot \nabla u_{h}=0$ on a type I triangle, and although this is not true on a type II triangle, this term appears with the right sign so its presence does not interfere with the basic stability result. As in the approach of Winther, another test function must be used to control the full norm on triangle boundaries.

\section{Analysis using characteristic coordinates}

There is another approach to error analysis of these methods which can be applied to other methods as well. In this approach, described more fully in [3], we use a coor- 
dinate system with one coordinate in the characteristic direction and a second coordinate either orthogonal to the characteristic direction or else lying along the inflow side of a type I triangle or the outflow side of a type II triangle.

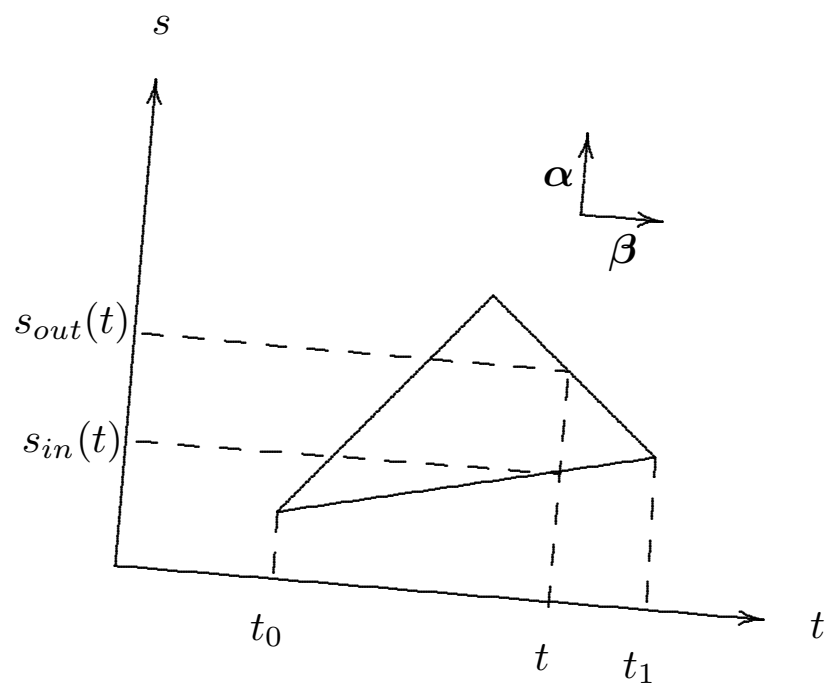

Fig. 1. Characteristic coordinates

In the notation of Fig. 1, a triangle $T$ may be described by

$$
T=\left\{(s, t): s \in\left[s_{\text {in }}(t), s_{\text {out }}(t)\right], t \in\left[t_{0}, t_{1}\right]\right\} .
$$

Using this coordinate system, one can integrate along the characteristics to write the exact solution in the form

$$
u(s, t)=u_{i n}(t)+\int_{s_{i n}(t)}^{s} f d s .
$$

We now show how to get a similar formula for the discontinuous Galerkin solution on a type I triangle. Since the function $s-s_{i n}(t)$ is linear and vanishes on $\Gamma_{i n}(T)$, $v_{h}=\left[s-s_{i n}(t)\right] q$ is a polynomial of degree $\leq n$ when $q$ is a polynomial of degree $\leq n-1$. Choosing this test function in (1), we get

$$
\left(\left[s-s_{i n}(t)\right]\left(u_{h}\right)_{s}, q\right)_{T}=\left(\left[s-s_{i n}(t)\right] f, q\right)_{T} .
$$

Since $\left(u_{h}\right)_{s} \in P_{n-1}(T)$ and $s-s_{i n}(t) \geq 0$ in $T$, this implies $\left(u_{h}\right)_{s}=R_{n-1} f$, where $R_{n-1}$ denotes the projection of $f$ into $P_{n-1}(T)$ with respect to the weighted $L^{2}$ inner product $[f, q]=\left(\left[s-s_{i n}(t)\right] f, q\right)$. Using this result, choosing $v_{h}=w(t)$ 
in (1), and rearranging terms, we also get

$$
\begin{aligned}
\int_{t_{0}}^{t_{1}}\left(u_{h, i n}^{+}-u_{h, \text { in }}^{-}\right) w(t) d t & =\left(f-R_{n-1} f, w\right)_{T} \\
& =\int_{t_{0}}^{t_{1}}\left[\int_{s_{\text {in }}(t)}^{s_{\text {out }}(t)}\left(f-R_{n-1} f\right)\right] w(t) d t .
\end{aligned}
$$

Since $u_{h, \text { in }}^{+}-u_{h, \text { in }}^{-} \in P_{n}\left[t_{0}, t_{1}\right]$, (polynomials of degree $\leq n$ in $t$ ),

$$
u_{h, \text { in }}^{+}-u_{h, \text { in }}^{-}=Q_{n} \int_{s_{\text {in }}(t)}^{s_{\text {out }}(t)}\left(f-R_{n-1} f\right) d s,
$$

where $Q_{n}$ denotes $L^{2}$ projection into $P_{n}\left[t_{0}, t_{1}\right]$. Hence

$$
\begin{aligned}
u_{h}(s, t) & =u_{h, i n}^{-}+\left[u_{h, i n}^{+}-u_{h, i n}^{-}\right]+\int_{s_{i n}(t)}^{s}\left(u_{h}\right)_{s} d s \\
& =u_{h, \text { in }}^{-}+Q_{n} \int_{s_{\text {in }}(t)}^{s_{\text {out }}(t)}\left(f-R_{n-1} f\right) d s+\int_{s_{\text {in }}(t)}^{s} R_{n-1} f d s .
\end{aligned}
$$

It follows immediately that

$$
\begin{aligned}
u\left(s_{\text {out }}(t), t\right)-u_{h}\left(s_{\text {out }}(t), t\right)=[ & \left.u_{\text {in }}-u_{h, \text { in }}^{-}\right] \\
& +\left(I-Q_{n}\right) \int_{s_{\text {in }}(t)}^{s_{\text {out }}(t)}\left(f-R_{n-1} f\right) d s .
\end{aligned}
$$

On a type II triangle, using the test function $v_{h}=w(t) \in P_{n}\left[t_{0}, t_{1}\right]$, we get by integration by parts that

$$
\begin{aligned}
(f, w)_{T} & =\left(\boldsymbol{\alpha} \cdot \nabla u_{h}, w\right)_{T}-\int_{\Gamma_{\text {in }}(T)} J\left[u_{h}\right] w \boldsymbol{\alpha} \cdot \boldsymbol{n} \\
& =\int_{\partial T} u_{h} w \boldsymbol{\alpha} \cdot \boldsymbol{n}-\int_{\Gamma_{\text {in }}(T)} J\left[u_{h}\right] w \boldsymbol{\alpha} \cdot \boldsymbol{n} \\
& =\int_{\Gamma_{\text {out }}(T)} u_{h}^{-} w \boldsymbol{\alpha} \cdot \boldsymbol{n}+\int_{\Gamma_{\text {in }}(T)} u_{h}^{-} w \boldsymbol{\alpha} \cdot \boldsymbol{n} \\
& =\int_{t_{0}}^{t_{1}}\left[u_{h, \text { out }}^{-}-u_{h, \text { in }}^{-}\right] w(t) d t .
\end{aligned}
$$

When $f=0$, since $u_{h, \text { out }}^{-}$and $w(t) \in P_{n}\left[t_{0}, t_{1}\right]$, we get that $u_{h, \text { out }}^{-}=Q_{n} u_{h, \text { in }}^{-}$.

One can then obtain an error analysis for this method by using $t$-dependent test functions. Setting $e=u-u_{h}$ and rewriting the discontinuous Galerkin error equation in the form

$0=\left(\boldsymbol{\alpha} \cdot \nabla e, v_{h}\right)_{T}-\int_{\Gamma_{\text {in }}(T)}\left[e^{+}-e^{-}\right] \boldsymbol{\alpha} \cdot \boldsymbol{n}=-\left(e, \boldsymbol{\alpha} \cdot \nabla v_{h}\right)_{T}+\int_{t_{0}}^{t_{1}}\left(e_{\text {out }}^{-}-e_{\text {in }}^{-}\right) v_{h} d t$, 
we get for test functions $v_{h}=v_{h}(t)$, that

$$
\int_{t_{0}}^{t_{1}}\left(e_{\text {out }}^{-}-e_{\text {in }}^{-}\right) v_{h} d t=0 .
$$

Choosing $v_{h}=Q_{n}\left(e_{\text {out }}^{-}+e_{\text {in }}^{-}\right)$, we get

$$
\left|Q_{n} e_{\text {out }}^{-}\right|^{2}=\left|Q_{n} e_{\text {in }}^{-}\right|^{2}
$$

Equivalently,

$$
\left|e_{\text {out }}^{-}\right|^{2}+\left|\left(I-Q_{n}\right) e_{\text {in }}^{-}\right|^{2}=\left|e_{\text {in }}^{-}\right|^{2}+\left|\left(I-Q_{n}\right) e_{\text {out }}^{-}\right|^{2} .
$$

On a type II triangle, $u_{h, \text { out }}^{-}$is a polynomial of degree $\leq n$ in $t$, so

$$
\left|\left(I-Q_{n}\right) e_{\text {out }}^{-}\right|=\left|\left(I-Q_{n}\right) u_{\text {out }}^{-}\right| \leq C h^{n+1 / 2}\|u\|_{n+1, T} .
$$

On a type I triangle, we get using (2) and the fact that $u_{h, \text { in }}^{-} \in P_{n}\left[t_{0}, t_{1}\right]$ that

$$
\left(I-Q_{n}\right) e_{\text {out }}^{-}=\left(I-Q_{n}\right) u_{\text {in }}+\left(I-Q_{n}\right) \int_{s_{\text {in }}(t)}^{s_{\text {out }}(t)}\left(f-R_{n-1} f\right) d s .
$$

Using this formula, it is not difficult to show that

$$
\left|\left(I-Q_{n}\right) e_{\text {out }}^{-}\right| \leq C h^{n+1 / 2}\|u\|_{n+1, T} .
$$

We can now sum these identities in the usual way to produce the standard error estimate

$$
\left|e_{\text {out }}^{-}\right|_{\Gamma_{\text {out }}(\Omega)}^{2} \leq\left|e_{\text {in }}^{-}\right|_{\Gamma_{\text {out }}(\Omega)}^{2}+C h^{2 n+1}\|u\|_{\Omega, n+1}^{2} \leq C h^{2 n+1}\|u\|_{\Omega, n+1}^{2} .
$$

A similar approach can be used to analyze the continuous Galerkin method. The test function $w(t)=Q_{n-1}\left(e_{\text {out }}^{\prime}+e_{i n}^{\prime}\right)$ produces the basic estimate

$$
\left|Q_{n-1} e_{\text {out }}^{\prime}\right|^{2}=\left|Q_{n-1} e_{i n}^{\prime}\right|^{2},
$$

from which an error estimate can be obtained using a similar technique. The analogue for the continuous Galerkin method of the condition $u_{h, \text { out }}^{-}=Q_{n} u_{h, \text { in }}^{-}$holding for the discontinuous Galerkin method on a type II triangle when $f=0$ is that $\left[u_{h, \text { out }}^{-}\right]^{\prime}=Q_{n-1}\left[u_{h, \text { in }}^{-}\right]^{\prime}$.

\section{A nonconforming piecewise linear approximation}

A natural question is whether this technique can be used to analyze methods that don't already have another method of error analysis. One class of such methods are those that lie somewhere between the continuous and discontinuous Galerkin methods in the sense that only certain moments are required to be continuous across element edges. Perhaps the simplest example is a method that seeks an approximate 
solution in the space of nonconforming piecewise linear elements, i.e., piecewise linear functions continuous at the midpoints of triangle edges. On a type II triangle, the solution would already be known at the midpoints of the two inflow edges (i.e., average values are continuous across edges). The remaining degree of freedom could be determined by requiring the finite volume condition that

$$
\int_{T} \boldsymbol{\alpha} \cdot \nabla u_{h} v=\int_{T} f v
$$

for all constant functions $v$. On a type I triangle, the solution would be known at the midpoint of the inflow edge and several possibilities exist for the remaining two equations. Perhaps the simplest is to require the finite volume condition above and also to require that the first moment be continuous across the inflow edge, i.e., continuity of $u_{h}$ across the inflow edge of a type I triangle. Again, it is not obvious how to analyze such a method since the test function $v=u_{h}$ is not allowed. An error analysis, using the $t$-dependent test functions described above, can be found in [1] in the case when each type II triangle, together with a type I triangle whose inflow side is the outflow side of the type II triangle, forms a parallelogram.

The identity for the homogeneous problem, which is the key to the analysis, is obtained by first integrating by parts to write the homogeneous variational equation in the form

$$
\int_{t_{0}}^{t_{1}}\left(u_{h, \text { out }}^{-}-u_{h, \text { in }}^{+}\right) v_{h} d t
$$

and choose the constant test function

$$
v_{h}=P_{\text {in }}\left(u_{h, \text { out }}+u_{h, i n}\right)
$$

on a type I triangle and

$$
v=P_{\text {out }}\left(u_{h, \text { out }}+u_{h, \text { in }}\right)
$$

on a type II triangle, where $P_{i n}$ is the $L^{2}$ projection into constant functions on $\Gamma_{i n}(T)$ on a type I triangle and $P_{\text {out }}$ is the $L^{2}$ projection into constant functions on $\Gamma_{\text {out }}(T)$ on a type II triangle. On a type I triangle, this leads to the identity

$$
\left|P_{\text {in }} u_{h, \text { out }}\right|^{2}=\left|P_{\text {in }} u_{h, \text { in }}\right|^{2}
$$

Since $P_{i n} P_{o u t}=P_{i n}$ on a type I triangle,

$$
\begin{aligned}
\left|P_{\text {out }} u_{h, \text { out }}\right|^{2} & =\left|P_{\text {in }} P_{\text {out }} u_{h, \text { out }}\right|^{2}+\left|\left(I-P_{\text {in }}\right) P_{\text {out }} u_{h, \text { out }}\right|^{2} \\
& =\left|P_{\text {in }} u_{h, \text { in }}\right|^{2}+\left|\left(I-P_{\text {in }}\right) P_{\text {out }} u_{h, \text { out }}\right|^{2} .
\end{aligned}
$$

On a type I triangle, it is easy to see that $P_{\text {out }} P_{\text {in }}=P_{\text {in }} P_{\text {out }}$ and since $\boldsymbol{\alpha} \cdot \nabla u_{h}=0$, $u_{h, \text { out }}(t)=u_{h, \text { in }}(t)$. Hence

$$
\left(I-P_{\text {in }}\right) P_{\text {out }} u_{h, \text { out }}=P_{\text {out }}\left(I-P_{\text {in }}\right) u_{h, \text { in }}=\left[d u_{h} / d t\right] P_{\text {out }} Q(t),
$$

where $Q(t)=t-t_{a}$, with $t_{a}$ being the average value of $t$ on $\left[t_{0}, t_{1}\right]$. Combining these results, we have on a type I triangle that

$$
\left|P_{\text {out }} u_{h, \text { out }}\right|^{2}=\left|P_{\text {in }} u_{h, \text { in }}\right|^{2}+\left[d u_{h} / d t\right]^{2}\left|P_{\text {out }} Q\right|^{2} .
$$


In a similar fashion, we can show on a type II triangle that

$$
\left|P_{\text {out }} u_{h, \text { out }}\right|^{2}=\left|P_{\text {out }} u_{h, \text { in }}\right|^{2}
$$

and then using the fact that $P_{\text {out }} P_{\text {in }}=P_{\text {out }}$, it follows that

$$
\begin{aligned}
\left|P_{\text {out }} u_{h, \text { out }}\right|^{2} & =\left|P_{\text {out }} u_{h, \text { in }}\right|^{2}=\left|P_{\text {out }} P_{\text {in }} u_{h, \text { in }}\right|^{2} \\
& =\left|P_{\text {in }} u_{h, \text { in }}\right|^{2}-\left|\left(I-P_{\text {out }}\right) P_{\text {in }} u_{h, \text { in }}\right|^{2} .
\end{aligned}
$$

Again, $P_{\text {out }} P_{\text {in }}=P_{\text {in }} P_{\text {out }}$, and since $\boldsymbol{\alpha} \cdot \nabla u_{h}=0, u_{h, \text { in }}(t)=u_{h, \text { out }}(t)$. Hence

$$
\left(I-P_{\text {out }}\right) P_{\text {in }} u_{h, \text { in }}=P_{\text {in }}\left(I-P_{\text {out }}\right) u_{h, \text { out }}=\left[d u_{h} / d t\right] P_{\text {in }} Q .
$$

Combining these results, we obtain

$$
\left|P_{\text {out }} u_{h, \text { out }}\right|^{2}+\left[d u_{h} / d t\right]^{2}\left|P_{\text {in }} Q\right|^{2}=\left|P_{\text {in }} u_{h, \text { in }}\right|^{2} .
$$

In the case when a type I triangle $T_{1}$ and a type II triangle $T_{2}$ form a parallelogram $P$, one can show that $\left|P_{\text {out }} Q\right|^{2}$ on $T_{1}$ is equal to $\left|P_{\text {in }} Q\right|^{2}$ on $T_{2}$. Since $\left[d u_{h} / d t\right]^{2}$ is continuous across the common boundary of $T_{1}$ and $T_{2}$, adding the above identities leads to cancellation of the $\left[d u_{h} / d t\right]^{2}$ terms and results in the identity

$$
\left.\left|P_{\text {out }} u_{h, \text { out }}\right|\right|_{\Gamma_{\text {out }}\left(T_{1}\right)} ^{2}=\left|P_{\text {in }} u_{h, \text { in }}\right|_{\Gamma_{\text {in }}\left(T_{2}\right)}^{2} .
$$

Since the above quantities are continuous across triangle edges, we may now sum over all triangles to produce a global stability result

$$
\left|P_{\text {out }} u_{h, \text { out }}\right|_{\Gamma_{\text {out }}(\Omega)}^{2}=\left|P_{\text {in }} u_{h, \text { in }}\right|_{\Gamma_{\text {in }}(\Omega)}^{2},
$$

which is analogous to the stability result for the continuous problem. An error analysis based on this approach leads to the estimate

$$
\left|P_{\text {out }}\left(u-u_{h}\right)\right|_{\Gamma_{\text {out }}(\Omega)}+\left\|u-u_{h}\right\|_{\Omega} \leq C h^{2}\|u\|_{3, \Omega} .
$$

Note that the estimate is of optimal order in $L^{2}$, but requires additional regularity of the solution. The key idea here is the fact that if the adjacent triangles $T_{1}$ and $T_{2}$ form a parallelogram and $u \in H^{3}\left(T_{1} \cup T_{2}\right)$, then for all $w \in P_{0}\left(T_{1} \cup T_{2}\right)$,

$$
\left|\int_{T_{1} \cup T_{2}} \boldsymbol{\alpha} \cdot \nabla\left(u-u_{I}\right) w d x d y\right| \leq C h^{2}\|u\|_{3, T_{1} \cup T_{2}}\|w\|_{0, T_{1} \cup T_{2}},
$$

where $u_{I}$ is the standard continuous piecewise linear interpolant of $u$.

\section{References}

1. Cai, D-M. : Reduced continuity finite element methods for hyperbolic equations. Ph.D. Dissertation, Rutgers University, (1991) 
2. Falk, R.S., Richter, G.R.: Analysis of a continuous finite element method for hyperbolic equations. SIAM J. Numer. Anal. 24 (1987) 257-278

3. Falk, R.S., Richter, G.R.: Local estimates for a finite element method for hyperbolic and convection-diffusion equations. SIAM J. Numer. Anal. 29 (1992) 730-754

4. Johnson, C., Pitkäranta, J.: An analysis of the discontinuous Galerkin method for a scalar hyperbolic equation. Math. Comp. 46 (1986) 1-26

5. Lesaint, P., Raviart, P-A.: On a finite element method for solving the neutron transport equation. Mathematical Aspects of Finite Elements in Partial Differential Equations, (C. de Boor, ed.), Academic Press, New York, (1974) 89-123

6. Reed, W. H., Hill, T. R.: Triangular mesh methods for the neutron transport equation. Los Alamos Scientific Laboratory Technical Report LA-UR-73-479 (1973)

7. Winther, R.: A stable finite element method for first-order hyperbolic systems. Math. Comp. 36 (1981) 65-86 